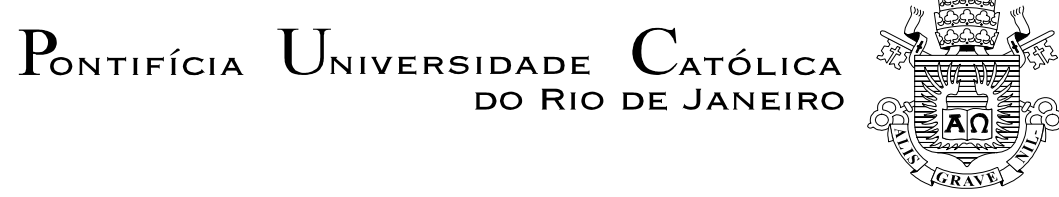

Tatiana Bagetti

Um Estudo Experimental do Processamento na Interface
Fônica e da Análise Sintática Inicial: O papel de Elementos
Funcionais na Aquisição da Linguagem

Tese de Doutorado

Tese apresentada ao Programa de Pós-graduação em Letras da PUC-Rio como parte dos requisitos parciais para obtenção do título de Doutor em Letras.

Orientadora Prof ${ }^{a}$. Letícia Maria Sicuro Corrêa

Rio de Janeiro

Abril de 2009 


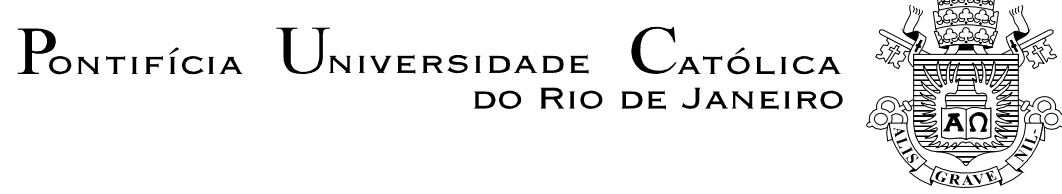

Tatiana Bagetti

\title{
Um Estudo Experimental do Processamento na Interface Fônica e da Análise Sintática Inicial: O papel de Elementos Funcionais na Aquisição da Linguagem
}

\begin{abstract}
Tese apresentada como requisito parcial para obtenção do grau de Doutor pelo Programa de Pós-Graduação em Letras do Departamento de Letras do Centro de Teologia e Ciências Humanas da PUC-Rio. Aprovada pela Comissão Examinadora abaixo assinada.
\end{abstract}

Profa. Letícia Maria Sicuro Corrêa

Orientadora

Departamento de Letras - PUC-Rio

Profa. Maria Cristina Lobo Name

UFJF

Profa. Marina Rosa Ana Augusto

Departamento de Letras - PUC-Rio

Profa. Raquel Santana Santos Santos

USP-SP

Profa. Carmen Lúcia Barreto Matzenauer

UCPEL

Prof. Paulo Fernando Carneiro de Andrade Coordenador Setorial do Centro de Teologia e Ciências Humanas - PUC-Rio.

Rio de Janeiro, , de de 
Todos os direitos reservados. É proibida a reprodução total ou parcial do trabalho sem autorização da universidade, da autora e da orientadora.

\section{Tatiana Bagetti}

Graduou-se em Fonoaudiologia pela Universidade Federal de Santa Maria (UFSM) em 2001 e concluiu, em 2003, o curso de Especialização em Fonoaudiologia (área de Linguagem) na mesma instituição. Obteve título de Mestre em Distúrbios da Comunicação Humana pela UFSM em 2005 e concluiu o Doutorado em Letras (área de concentração- Estudos da Linguagem) pela Pontifícia Universidade Católica do Rio de Janeiro (PUC-Rio) em 2009. Atua, desde 2005, no Grupo de Pesquisas do LAPAL - Laboratório de Psicolingüística e Aquisição da Linguagem da PUC-Rio. Áreas de interesse: Psicolingüística, Aquisição da Linguagem, Fonoaudiologia, Déficits da linguagem, desvio fonológico.

Ficha Catalográfica

\section{Bagetti, Tatiana}

Um estudo experimental do processamento na interface fônica e da análise sintática inicial: o papel de elementos funcionais na aquisição da linguagem / Tatiana Bagetti; orientadora: Letícia Maria Sicuro Corrêa. - 2009.

151 f. ; il. (col.) ; $30 \mathrm{~cm}$

Tese (Doutorado em Letras)-Pontifícia Universidade Católica do Rio de Janeiro, Rio de Janeiro, 2009.

Inclui bibliografia

1. Letras - Teses. 2. Aquisição da linguagem. 3. Desencadeamento fonológico. 4. Categorias funcionais. 5. Determinantes. 6. Afixos verbais. I. Corrêa, Letícia Maria Sicuro. II. Pontifícia Universidade Católica do Rio de Janeiro. Departamento de Letras. III. Título. 
A minha mãe, Lúcia, pelo coração e ao meu pai, Vilmar, pela razão. 


\section{Agradecimentos}

À minha orientadora Letícia Maria Sicuro Corrêa, pela orientação constante e cuidadosa, pelo exemplo de dedicação à pesquisa, pela sabedoria, bom-humor, carinho e principalmente pelo amor contagiante pela pesquisa em aquisição da linguagem.

A todos os colegas do LAPAL, especialmente a Mercedes, Luciana, Clara, René, Jaqueline, Ferrari, Erica e Marina pelos momentos agradáveis compartilhados, pelas trocas e companheirismo nesta caminhada.

Ao Claver pelo apoio técnico no babylab e a Ingrid, Marcos, Lúcia e Roberta pela disposição, eficiência e auxílio na aplicação dos experimentos.

A todas as crianças testadas e aos pais das crianças que levaram seus filhos ao LAPAL e também àqueles que, mesmo sem ter ido ao LAPAL, gentilmente, autorizaram a participação dos seus filhos nos experimentos realizados nas creches.

Às escolas Santa Terezinha, Tabladinho e à creche Pirilampos pela atenção, receptividade e pelo espaço físico disponibilizado para a realização dos experimentos.

A Professora Raquel Santana Santos por gentilmente receber-me como aluna ouvinte na USP. Obrigada pela acolhida e pelos ensinamentos transmitidos!

Às professoras Carmen Matzenauer, Marina Rosa Ana Augusto, Érica Rodrigues, Raquel Santana Santos e ao professor Ricardo Joseh Lima por terem gentilmente aceitado fazer parte da minha banca de doutorado e pela leitura cuidadosa e enriquecedora da Tese.

À $P U C$ e ao $C N P q$, pela bolsa de doutorado a mim concedida.

Aos meus pais, pelo amor incondicional, pelo porto-seguro, força constante, apoio e pelos esforços sem medida empreendidos em minha formação como ser 
humano.

Aos meus irmãos Aline, Milena e Mateus que, apesar de distantes, sempre estiveram presentes. Obrigada pelo apoio, carinho, amizade, e pelo afeto constantes!

Ao Márcio Vieira Correia pelo amor, incentivo, força e por poder compartilhar comigo os momentos alegres e também os mais difíceis. Obrigada, também, por me emprestar sua família (Analice, Batista, Michele, Michel, Mariana e Alex), que agora também é minha!

A Daniele Fialho e Carla Santos, pelo carinho, incentivo, segurança e principalmente pela amizade verdadeira.

À Vanessa Gouveia, que "emprestou-me" sua voz para a elaboração dos experimentos e por ter se tornado uma grande amiga; sempre presente em todos os momentos.

Aos meus amigos, Cláudia Paz, Renata Santos, Paulinho Mota, Márcia Brito, Augusto, Virpi Turumen, Raul e Vanessa Campos, pelo carinho, confiança e pelos momentos agradáveis compartilhados.

A Daisaku Ikeda, pelo grande exemplo de pessoa que és, e aos meus companheiros budistas, especialmente Ana Carla, Karina e Renata, pelo incentivo constante e por acreditarem sempre em mim e terem contribuído com o meu crescimento pessoal.

A todas as pessoas que de alguma forma contribuíram com a realização deste trabalho. 


\section{Resumo}

Bagetti, Tatiana; Corrêa, Letícia Maria Sicuro (Orientadora). Um estudo experimental do processamento na interface fônica e da análise sintática inicial: o papel de elementos funcionais na aquisição da linguagem. Rio de Janeiro, 2009. 151p. Tese de Doutorado Departamento de Letras, Pontifícia Universidade Católica do Rio de Janeiro.

Este estudo tem como foco a passagem da percepção fônica para a representação morfofonológica de elementos funcionais, mais precisamente os afixos verbais, bem como a realização do parsing lingüístico no processo inicial de aquisição da linguagem. Nesta tese a aquisição da linguagem é abordada em uma perspectiva psicolingüística de forma integrada com a Teoria Lingüística Gerativa, em sua versão Minimalista (Corrêa, 2006). A hipótese que orienta este trabalho é a de que os elementos de classe fechada são percebidos pela criança de forma diferenciada e que há uma distinção entre a percepção inicial desses elementos no nível fonético/fonológico e a sua posterior representação morfofonológica. Esses elementos, uma vez representados em termos de categorias funcionais do léxico, contribuem para a realização do parsing lingüístico pela criança. Uma análise de histórias infantis demonstrou que determinantes e afixos tendem a ocorrer em fronteiras de frases fonológicas e que propriedades fonéticas, como o acento, podem favorecer a percepção inicial de afixos verbais. Foram conduzidos três experimentos, sendo que os dois primeiros fizeram uso da Técnica de Escuta Preferencial e o terceiro utilizou a Técnica de Fixação Preferencial do Olhar. O primeiro experimento teve como objetivo avaliar a sensibilidade das crianças (de 9 a 15 meses) adquirindo o Português Brasileiro a distinções fônicas que afetam o padrão silábico da língua, independentemente do ambiente morfológico em que estas ocorrem (afixos flexionais e raízes de Nomes). O segundo experimento visou a verificar se o ambiente morfológico (afixos flexionais e raízes de Nomes) afeta o modo como crianças (de 9 a 18 meses) adquirindo o PB percebem alterações fônicas que não afetam o padrão silábico, o que indicaria que estas percebem afixos verbais como uma classe morfológica. O terceiro experimento pretendeu verificar se as crianças com idades entre 17 e 23 meses realizam o parsing de enunciados lingüísticos, diferenciando elementos lexicais homófonos em função da natureza de elementos funcionais. Os resultados sugerem que crianças com média de 11 
meses são capazes de perceber alterações fônicas independentemente do ambiente morfofonológico em que estas ocorrem. Também foi constatado que crianças entre 9 a 12 meses percebem alterações fônicas que não afetam o padrão silábico da língua em elementos de classe fechada, sugerindo que esses elementos são percebidos pelas crianças como uma classe morfológica em uma fase inicial no processo de aquisição da linguagem. Além disso, foi verificado que as crianças com média de 21 meses respondem diferencialmente a palavras homófonas em classes gramaticais diferentes (Nome e Verbo), com base na distinção entre projeções mínimas e máximas do Determinante, no parsing lingüístico. A marcação do afixo verbal não afeta o reconhecimento do verbo, mas formas marcadas em relação a tempo adicionam dificuldade à condução da tarefa. Os resultados encontrados são compatíveis com as hipóteses testadas e permitem que se reconstrua a passagem da percepção do estímulo lingüístico em um nível fônico para a representação morfofonológica de elementos de classe fechada, e finalmente para a representação dos mesmos como elementos de categorias funcionais, os quais são essenciais na condução do parsing lingüístico.

\section{Palavras-chave}

Aquisição da Linguagem; desencadeamento (bootstrapping) fonológico; categorias funcionais; Determinantes; afixos verbais; parsing 


\section{Abstract}

Bagetti, Tatiana; Corrêa, Letícia Maria Sicuro (Advisor). An experimental study of the early processing at the phonetic inteface and the early parsing in language acquisition: the role of functional elements. Rio de Janeiro, 2009. 151p. Doctoral Thesis - Departamento de Letras, Pontifícia Universidade Católica do Rio de Janeiro.

This study focuses on the passage from speech perception to the morphophonological representation of functional elements, verbal affixes in particular, and on the early parsing of linguistic utterances in language acquisition. A psycholinguistic perspective to language acquisition is adopted together with a minimalist conception of language (Corrêa, 2006). The working hypothesis is that closed class elements are distinctively perceived by children initially at a phonetic/phonological level and subsequently at a morphophonological one. Their representation as functional elements at a later stage contributes to the parsing of linguistic utterances. An analysis of a set of tales for children has demonstrated that determiners and verbal affixes occur at the edges of phonological phrases and phonetic properties such as the accent may contribute to their early perception by children. Three experiments were conducted, the first two in Head-turn Paradigm and the latter in the Intermodal Preferential Looking paradigm. Experiment 1 aimed at assessing 9-15 month infants' sensibility to phonetic distinctions in the linguistic stimulus, which affect the syllabic pattern of the language (Brazilian Portuguese), independently of the morphological context in which they occur (verbal affixes and nominal roots). Experiment 2 aimed at verifying whether these morphological contexts affect infants' perception of phonetic alterations that do not affect the phonological pattern of the language. The perception of such distinctions in the verbal affixes, but not in the nominal roots, was considered to indicate sensibility to the morphophonological patterns of these closed class elements. The third experiment aimed at verifying the extent to which children by the age of 21 months would rely on functional information in the parsing of linguistic utterances, thereby ascribing different categorical features to homophonous words (nouns and verbs). The results of Experiment 1 suggest that 9-15 month infants do perceive phonetic alterations that affect the syllabic pattern of language, regardless of the morphological context in which they occur. The results of the 
Experiment 2 suggest that infants are sensitive to phonetic alterations that do not affect the syllabic pattern of the language by the end of their first year of life (9-12 months). The results of Experiment 3 suggest that take into account different syntactic projections of the determiner in ascribing homophonous words to different classes (noun and verb). These results also indicate that verbs are analyzed as such regardless of the type of morphological affix they present (marked or unmarked for Tense). However, tensed marked forms seem to add processing costs in the accomplishment of the task. These results are compatible with the hypotheses that guided the present thesis and enable a theory of language acquisition to reconstruct the passage from the phonetic perception of the linguistic stimulus to the morphophonological representation of closed class elements (verbal affixes) and from this level of representation to children's reliance on functional elements in the parsing of linguistic utterances.

\section{Keywords}

Language acquisition; phonological bootstrapping; functional categories; determiner; verbal affixes; parsing 


\section{Sumário}

1 Introdução

2 Arcabouço teórico: uma integração entre bootstrapping fonológico e o Programa Minimalista

2.1 O problema do bootstrapping e a hipótese do bootstrapping fonológico

2.2 A percepção de pistas fonéticas/fonológicas e prosódicas relevantes para o processamento sintático pelo bebê

2.3 A integração do bootstrapping fonológico com o Programa Minimalista - o bootstrapping da sintaxe

3 Elementos funcionais: sua disponibilidade, evidências de seu processamento e habilidades iniciais de parsing

3.1 Controvérsias sobre a disponibilidade de categorias funcionais

3.2 A teoria da fonologia prosódica e sua relação com a identificação dos elementos funcionais

3.3 Evidências do processamento de elementos funcionais por bebês

3.4 Estudos sobre a aquisição da ordem das palavras e a habilidade inicial de parsing

4 Metodologia Experimental

4.1 Técnica de Escuta Preferencial

4.1.1 Descrição da Técnica Original

4.1.2 Descrição da Técnica e procedimentos realizados no LAPAL

4.2.2 Descrição da Adaptação da Técnica de Fixação 
Preferencial

5 Experimentos em Escuta Preferencial 78

$\begin{array}{ll}5.1 \text { Experimento } 1 & 78\end{array}$

$\begin{array}{ll}5.2 \text { Experimento } 2 & 88\end{array}$

6 Experimentos em Fixação Preferencial do Olhar 100

7 Conclusões 114

8 Referências bibliográficas 118

9 Anexos 131

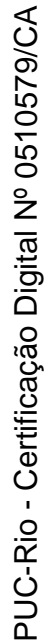




\section{Listas de Quadros}

Quadro 1 - Distribuição dos determinantes nas fronteiras dos constituintes prosódicos

Quadro 2 - Distribuição dos afixos verbais nas fronteiras dos constituintes prosódicos

Quadro 3 - Alterações fônicas em seqüências tônicas de afixos flexionais em posição de Rima final (final de sílaba, final de palavra) e em radicais de nomes em posição de Rima medial (final de sílaba, dentro de palavra) e final Quadro 4 - Tempo médio (em seg.) por criança e por 87 condição $(n=10)$

Quadro 5 - Alterações fônicas que não afetam o padrão silábico da língua em seqüências tônicas de afixos flexionais em radicais de nomes em posição de rima final (final sílaba, final de palavra) e em radicais de nomes em posição de Rima medial (final de sílaba, dentro de palavra) e final Quadro 6 - Tempo médio de escuta (em seg.) por criança e por condição $(n=21)$

Quadro 7 - Tempo médio de escuta das crianças nos grupos G1 e G2

Quadro 8 - Consistência da fixação do olhar das crianças para as imagens-alvo nas condições Nome, V1 e V2 


\section{Listas de Gráficos}

Gráfico 1 - Percentagem de determinantes nas fronteiras dos constituintes prosódicos $(n=216)$

Gráfico 2 - Percentagem de afixos verbais nas fronteiras dos constituintes prosódicos $(\mathrm{n}=200)$

Gráfico 3 - Tempo médio de escuta por condição $(n=10)$

Gráfico 4 - Tempo médio de escuta por condição $(n=21)$

Gráfico 5 - Tempo médio de escuta das crianças nos grupos G1

(9 a 12 meses) e do grupo G2 (14 a 18 meses)

Gráfico 6 - Média do tempo de fixação do olhar (cs) para a imagem correspondente ao Nome em função da categoria da palavra crítica no estímulo acústico

Gráfico 7 - Média do tempo de fixação do olhar (cs) para a imagem correspondente ao Verbo em função da categoria da palavra crítica no estímulo acústico

Gráfico 8 - Média do tempo de fixação do olhar (cs) para a imagem correspondente ao Verbo em função da categoria da palavra crítica no estímulo acústico

Gráfico 9 - Média do tempo de fixação do olhar (cs) para a imagem correspondente ao Verbo em função da categoria da palavra crítica no estímulo acústico 


\section{Listas de Figuras}

Figura 1 - Esquema da Técnica de Fixação Preferencial do

Olhar

Figura 2 - Imagem dos equipamentos utilizados na adaptação da Técnica de Fixação Preferencial do Olhar

Figura 3 - Exemplo de estímulos apresentados durante a fase de teste do experimento 3 